\title{
Atención médica de urgencia: organización para la recepción de víctimas múltiples*
}

\author{
Emergency care: organization for receiving many victims
}

\author{
Víctor Rene Navarro Machado'; Marcos D. I raola Ferrer'"; Gabriel Rodríguez \\ Suárez'; Arelys Falcón Hernández'"'; Belkys Rodríguez Llerena'"I \\ 'Especialista de II Grado en Medicina Intensiva y Emergencias. Asistente. Hospital \\ General Universitario "Dr. Gustavo Aldereguía Lima". Cienfuegos, Cuba. \\ "Especialista de II Grado en Medicina Intensiva y Emergencias. Hospital General \\ Universitario "Dr. Gustavo Aldereguía Lima". Cienfuegos, Cuba. \\ "IEspecialista de II Grado en Medicina Intensiva y Emergencias. Instructor. Hospital \\ General Universitario "Dr. Gustavo Aldereguía Lima". Cienfuegos, Cuba.
}

\section{RESUMEN}

Introducción La importancia de estar preparados para enfrentar situaciones de desastres cobra mayor relevancia para los servicios de urgencia y especialmente cuando el evento ocurre de forma inesperada.

Objetivos Debatir las acciones a desarrollar para el manejo de eventos con víctimas múltiples, principalmente en los de inicio súbito, lo que permitiría perfeccionar los planes ante situaciones de desastres. En segundo lugar, someter a la consideración de especialistas un "sistema de tarjetas de funciones" para estas situaciones, diseñado para los servicios de urgencia de las principales instituciones de salud del territorio.

Métodos Desarrollo de un taller con participación de profesionales seleccionados por su experiencia en la organización y el trabajo en los servicios de urgencia. Luego de dos conferencias plenarias, el grupo se dividió en dos comisiones, la primera trabajó los aspectos de organización prehospitalaria y la segunda en la hospitalaria. Finalmente, ambos grupos se unieron para exponer sus recomendaciones.

Conclusiones Es necesario generar encuentros similares para incrementar el intercambio entre las instituciones, los decisores y profesionales encargados del estudio y la preparación del sistema de salud ante desastres. El tema debe extenderse al proceso de capacitación tanto a pre como a posgrado; se necesita de 
algunos recursos para una respuesta más efectiva, en especial medios de comunicación y para la logística del escenario; el "sistema de tarjetas de funciones", aprobado por todos, sirve metodológicamente a la respuesta, primero en función de la actualización permanente del personal y segundo por constituir una versión resumida del plan de estas instituciones.

Palabras clave: Atención médica de urgencia, desastres, Cuba.

\begin{abstract}
S
I ntroduction The importance of being prepared to face disasters is greater for the emergency service and particularly when unexpected events occur.

Objectives To discuss the actions to be taken to face disaster events with many victims, mainly those that suddenly occur, which will allow improving plans for disasters, and also to submit a "system of function cards" to experts, which was designed for the emergency services of the main health institutions in the territory. Methods A workshop with the involvement of selected experienced professionals in the organization and operation of emergency services was held. After two plenary sessions, the group was divided into two commissions, the first one discussed aspects of pre-hospital organization and the second one analyzed hospital care. Finally, both groups met to make their recommendations.

Conclusions It is necessary to promote meetings similar to this workshop in order to increase the exchange of ideas among the institutions, the decision-makers and the professionals in charge of the study and preparation of the healthcare system for disaster management. This topic should be included in the training process at both undergraduate and graduate educational levels. Some additional resources for a more effective response, particularly means of communication and scenario logistics are required. The approved "system of function cards" methodologically serves to give response, firstly in terms of the permanent staff updating and secondly, because this system is a summarized version of the disaster plans of these institutions.
\end{abstract}

Key words: Emergency care service, disasters, Cuba.

\title{
I NTRODUCCI ÓN
}

Es conocida la relevancia para los servicios de salud que tienen los desastres y en especial para los servicios de urgencia; típicamente, aún antes del impacto de estos fenómenos, tienden a ocurrir problemas específicos que involucran al sector de la salud; ya desde la fase informativa, se producen lesiones físicas o psíquicas que requieren de una atención médica inmediata y el primer aspecto a medir tras un desastre es el número de fallecidos, lesionados o enfermos, con independencia de que en un segundo tiempo, comiencen a aparecer un grupo de afecciones de inicio lento en aquellas áreas donde están presentes el hacinamiento y el saneamiento deficiente. 
Para los sistemas de urgencia, el enfrentamiento a los desastres representa un reto, en especial porque no todos se presentan de igual forma y en los de inicio súbito, son los primeros en recibir las víctimas o salir al escenario donde la situación es muchas veces confusa y carente de recursos. Por ello, si bien su preparación es decisiva, el enfoque de la respuesta, que debe ser multisectorial, incluye una serie de principios que se inician con el proceso de alerta y la activación del plan de aviso, a lo que siguen las actividades en el lugar del siniestro, el enlace de las instituciones prehospitalaria y hospitalaria, el manejo de las evacuaciones primarias y secundarias y la recepción de las víctimas en los centros de salud.

Ante cualquier tipo de desastre, las primeras horas son realmente críticas. El pánico cunde de inmediato en el ambiente, la población se encuentra desorientada y no sabe en principio qué hacer ni cómo organizarse, a pesar incluso de haber recibido adiestramiento previo. La asistencia médica en situaciones de desastre tiene como objetivo principal garantizar la atención médica preventiva, curativa de los lesionados o enfermos, el aseguramiento de las medidas higiénico-epidemiológicas y la evacuación médica organizada en caso necesario de los lesionados o enfermos que se produzcan en la población, empleando de la forma más racional y efectiva los recursos de la red preventiva asistencial que existe en el país, siguiendo el principio de territorialidad bajo la dirección y el control de los Consejos de Defensa en cada instancia. A la vez que garantiza la reducción de la vulnerabilidad en sus instituciones.

El taller realizado tuvo dos objetivos centrales, en primer lugar debatir las acciones a desarrollar para el manejo de eventos con víctimas múltiples, principalmente de inicio súbito; lo que permitiría perfeccionar los planes para este tipo de situación. El segundo, fue someter a la consideración de los invitados, para su

perfeccionamiento, una visión panorámica de un "sistema de tarjetas de funciones", desarrollado desde el año 2001 en el Hospital General Universitario "Dr. Gustavo Aldereguía Lima" de Cienfuegos y que basados en la experiencia previa en este hospital, se diseñó para su generalización a todos los servicios de urgencia de los policlínicos, la emergencia médica móvil (EMM) y el resto de los hospitales de la provincia.

\section{MÉTODOS}

El taller fue organizado por los relatores de este informe, con especialistas del territorio y algunos expertos invitados de territorios cercanos; todos con experiencia en el trabajo de organización de los servicios de urgencia tanto prehospitalarios como hospitalarios. Participaron además profesionales de la dirección del Sistema Integrado de Urgencias Médicas (SIUM), directores de hospitales y miembros de sus consejos de dirección así como médicos y otros profesionales que trabajan en los servicios de urgencia de todos los policlínicos de la provincia de Cienfuegos.

El evento se inició con dos conferencias plenarias sobre el tema de organización de la asistencia médica en desastres súbitos, que incluyó los ejes temáticos para la posterior discusión.

Luego los participantes se dividieron en dos comisiones presididas por un moderador auxiliado por un relator. La primera trabajó los aspectos de organización del escenario y de los servicios de urgencia en policlínicos y la segunda en la organización en los servicios de urgencia hospitalarios. Los organizadores asignaron 
los participantes a cada grupo según especialidad y experiencia, asegurando el equilibrio de la actividad.

Para cada una de las comisiones se preparó, con la participación de especialistas consultados, una guía temática para orientar la discusión de los grupos de trabajo y hacer sus discusiones más productivas; también con la intención de facilitar la labor de los moderadores y relatores.

En total el evento contó con 35 participantes, de los cuales 18 llevaban más de 10 años dedicados a la atención del paciente grave incluida la preparación de sus servicios para la recepción de víctimas múltiples, 6 ostentaban grados científicos y 15 eran especialistas de II Grado principalmente en medicina intensiva y emergencias.

Al final de cada sesión, fue presentada la propuesta del "sistema de tarjetas de funciones" para situaciones de desastres, ya citado. Este sistema se extenderá ahora a las principales instituciones de salud del territorio; fue valorado, enriquecido y aprobado, primero en cada comisión por separado y luego por todos. Una vez conluido el debate, se procedió a la discusión del relato por el grupo antes de presentarlo en sesión plenaria para su debate final y aprobación.

La sesión de discusión se organizó alrededor de seis ejes temáticos:

- Desastres de inicio súbito.

- Atención medica de emergencia.

- Organización en el escenario.

- Organización de la emergencia médica móvil.

- Organización de los servicios de urgencia prehospitalarios.

- Organización de los servicios de urgencia hospitalarios.

Para cada uno de estos acápites se preparó una guía de preguntas o temas orientados a ordenar la discusión de los grupos de trabajo y hacer sus discusiones más productivas. El grupo de moderadores y relatores se reunió previamente al taller para examinar los objetivos, aspectos metodológicos y examinar críticamente los temas a debatir.

El trabajo de cada comisión se inició con una introducción general sobre el tema, los objetivos a perseguir, los subtemas a ser tratados que estuvieron incluidos en las preguntas formuladas y otros planteados por los integrantes de cada grupo. Los moderadores y relatores fueron seleccionados por su amplio conocimiento del tema, experiencia de trabajo en los servicios de urgencia, prestigio y autoridad.

Comisión 1: servicios de urgencia extrahospitalarios

Preguntas problemas para la discusión

1. ¿Cuáles aspectos o elementos que pudieran limitar un rápido despacho?

2. ¿Se envían escalonadamente los recursos o se espera por la llegada del primer móvil? 
3. ¿J efe el puesto médico en el escenario?

4. ¿Con quién y con qué se organiza el puesto médico de avanzada?

5. ¿Brigadas médicas?

6. ¿Incremento de ambulancias?

7. ¿Logística para el escenario?

8. ¿Sistema de señalización?

9. Evacuación controlada ¿Cómo?

10. ¿Registros médicos? Nuestra propuesta.

11. ¿Qué nos falta en la preparación y capacitación?

\section{Respuestas de la comisión 1 sobre las correspondientes preguntas problemas}

1. Se consideraron como limitantes de un rápido despacho el conocimiento parcial del problema real al inicio, sobre todo en un evento súbito de múltiples heridos; las dificultades en la comunicación, principalmente con las ambulancias y la disponibilidad de transporte sanitario con que cuente el centro coordinador o las bases de ambulancias en el momento del evento.

2. En relación con el envió de ambulancias al lugar de escenario se considero oportuno tener en cuenta la información preliminar y después la retroalimentación con los primeros equipos de rescate que lleguen al sitio del desastre. No se debe enviar los recursos que no son necesarios.

3. El jefe del puesto de mando del escenario, centro de la toma de decisiones, debe ser una persona de amplios conocimientos tanto de organización ante desastres como del territorio donde se produce este. En las instituciones como por ejemplo un aeropuerto, es su director el primero en llegar, comienza el trabajo organizativo y entrega el mando a los representantes de la Defensa Civil (jefe de la zona de defensa), los cuales dirigirán el proceso según sus escalas de mando.

4. Se destaca la importancia del puesto médico de avanzada, que es el que organiza la atención en el escenario y que muchas veces constituye la primera tripulación sanitaria que arriba al escenario. La experiencia indica que las ambulancias deben partir de sus bases con tripulación extra (un médico y uno o dos enfermeros) y maletas de reanimación adicionales, la ambulancia regresa mientras el personal extra trabaja en función de la atención médica de los que no pueden transportarse.

Hay poco personal entrenado en triar en especial para el prehospitalario, por lo cual se

deben diseñar cursos preferentemente de corta duración, que lo contemplen. 
Los protocolos para la implementación de la atención en el escenario no suelen estar

sometidos a práctica previa, en especial como un sistema intersectorial. La solución es el

trabajo y la ejercitación conjunta en simulacros y ejercicios.

5. Se consideró de vital importancia que las instituciones de salud tengan organizadas brigadas médicas, que reciban capacitación y entrenamiento sistemático. Las brigadas médicas no se improvisan. Necesitan para ser efectivas estar listas en un tiempo mínimo (30 min). No se recomienda para hospitales pequeños disponer de sus especialistas para ello, principalmente si serán el destino final de la recepción de las víctimas.

Se señala que en los grandes desastres las brigadas deben ser multidisciplinarias donde esten presentes ingenieros, electricistas, arquitectos y otros, pues es este personal el que garantizará la seguridad de la escena para que el personal sanitario comience su labor. Se hace énfasis en la importancia de la intersectorialidad.

6. No hay suficientes ambulancias equipadas para transporte seguro y eficaz de los lesionados, ante esta situación se plantea la conversión de los vehículos acordados a través de la Defensa Civil y la utilización de transporte convencional para los códigos verdes, con lo cual las ambulancias se designan sólo para los casos amarillo o rojo.

Se consideró la posibilidad de que un grupo de pacientes lesionados leves puedan trasladarse en transporte no sanitario, pero siempre a un centro de salud donde puedan reevaluarse y definir que no han cambiado su clasificación inicial.

7. Otro importante tema abordado fue el apoyo logístico en el sitio del escenario donde deben garantizarse tiendas de campañas, planta eléctrica, medios de iluminación manual como linternas y vestimenta adecuada para el personal que trabajará en estos sitios. Es decisivo disponer de medios de comunicación para mantener la vitalidad del puesto médico de avanzada y del puesto de mando del escenario.

Para situaciones difíciles, en especial oscuridad y lluvia, es necesario disponer de recursos que permitan la vitalidad de la organización del escenario, ello debe ser organizado con los recursos propios pues las demandas no podrían realizársele a terceros que no tienen responsabilidad de garantizárnoslo.

Se consideró como muy importante tener preparados previamente paquetes para la atención de determina cantidad de víctimas. Para la EMM, es decisivo disponer de recursos propios para el reavituallamiento de las ambulancias, pues obtenerlos de los servicios de urgencia podría hacer más complicado el trabajo de ambos.

8. Es necesario organizar un sistema de señales que permita tanto la organización de las zonas de trabajo como reconocer rápidamente los principales jefes, los puestos de trabajo del escenario y las vías de comunicación.

El sistema para el triage debe ser sencillo, el mismo desde el escenario hasta las unidades de cuidados intensivos. 
9. La evacuación debe hacerse hacia la institución con capacidad resolutiva según el caso, esta debe ser informada y el paciente debe entregarse de forma individualizada. No se recomienda trasladar sin haber antes evaluado.

10. En el plan tanto prehospitalario como de las instituciones de salud deben estar definidas las tareas de cada persona, en tal sentido, el sistema por tarjetas de funciones es una buena opción. Fue de total aceptación la propuesta de tarjetas en los policlínicos y se sugirió que están deben estar colocadas en sitios bien visibles.

Se presenta y aprueba la propuesta de historia clínica (no existe similar en el país) para el manejo prehospitalario en situaciones de desastres, aunque se le debe añadir un acápite para los quemados (anexo).

11. En la actualidad los diferentes servicios de salud e instituciones tienen planes para el enfrentamiento de los desastres que no son uniformes y esto es un aspecto negativo cuando se presenta una situación de desastre real.

Las provincias deben hacer estudios de riesgos para poder evaluar las zonas más vulnerables y trabajar en la capacitación del personal que vive o trabaja en estas zonas.

La docencia médica actual no contempla en toda su magnitud la preparación de los estudiantes que después serán los futuros profesionales encargados de enfrentar los diferentes desastres.

EI SIUM tiene la responsabilidad de participar en la capacitación de todo el personal que interviene durante un desastre (en especial en los conceptos de apoyo vital), incluidos socorristas tanto de grupos especiales como la policía, rescate y salvamento, como de la población en general.

Debemos llegar a la población a través de los medios de difusión y aprovechar los telecentros para impartir temas relacionados con el socorrismo y con el manejo de víctimas múltiples. Cuando una institución hace su plan de reducción de los efectos de desastres debe tener en cuenta los diferentes sectores que deben cooperar con ella ante una situación de este tipo.

Comisión 2: servicios de urgencia hospitalarios

Preguntas problemas para la discusión

1. ¿Estamos preparados?

1. ¿Para cualquier evento? ¿Inter e intrasectorialidad?

2. ¿Bases para el grado de respuesta?

3. ¿Quién clasifica? ¿Sólo una persona?

4. ¿Organización siempre por códigos? ¿Estrategias para la asistencia médica?

5. ¿Evacuación y expansión?

6. ¿Logística y su organización? 


\section{Respuestas de la comisión 2 sobre las correspondientes preguntas problemas}

1. Aunque en nuestra provincia hay avances, es necesario realizar ejercicios conjuntos con todos los integrantes del sistema de salud, ello mejora la cooperación y el conocimiento de cada uno de la tarea que le toca y como integrarse para una mejor respuesta. Los accidentes con avisos previos han permitido una correcta preparación del personal para la recepción, pero es necesario reducir al mínimo los eventos en los cuales los pacientes llegan primero que la información, ello pasa principalmente en el municipio cabecera.

La activación de planes y recursos debe incluir a todos los hospitales del territorio, muchas veces la información a los hospitales pediátricos llega incompleta e incluso no se les informa el cese de la fase de alarma.

Para la organización ante desastres químicos, se debe mantener una constante actualización de los botiquines antitóxicos y una estrecha relación con los grupos toxicológicos de cada provincia y el Centro Nacional de Toxicología (CENATOX).

Es importante clarificar y convenir lo que se necesita de la policía en función de la protección del centro, cuidado de valores y pertenencias, así como la organización de la circulación vial.

Su función debe estar incluida en las tarjetas de funciones.

2. Las bases para el grado de respuesta: activación parcial o total del plan, recursos, liberación de camas, entre otros, dependerá de varios aspectos entre los que se destacan: número de casos, principalmente código rojo (personas con riesgo inminente para la vida), recursos humanos, tipo de institución y recursos materiales en especial ventiladores pulmonares y quirófanos. Este especto se debe discutir con los jefes de guardia, posterior a lo cual estará implícito en el proceso de capacitación.

3. El problema del arribo de niños a hospitales de adultos se ha resuelto de dos formas, la primera es luego de clasificarlos y solucionar las condiciones que implican riesgo vital, enviarlos en una ambulancia especializada al hospital pediátrico que tenga condiciones para recibirlos previa coordinación y la segunda es que especialistas de estas instituciones vayan al hospital receptor a realizar las intervenciones necesarias. En cualquier situación, se recomienda mantener recursos adecuados en todos los servicios de urgencia principalmente para los pacientes más pequeños.

El área de clasificación debe estar dirigida por la persona más preparada, pero en hospitales pequeños con pocos cirujanos, no se les recomienda dado la importante tarea de definir e intervenir inmediatamente los casos rojos que precisan de cirugía.

Según algunas experiencias, se debe designar en la entrega de guardia vespertina la persona que participará en la clasificación de los pacientes teniendo en cuenta sus conocimientos y jerarquía dentro de la institución.

4. El sistema de clasificación deberá ser sencillo y aplicado por todo el sistema. En nuestra experiencia, la utilización del código por colores, aunque la sensibilidad y especificidad dependen mucho del clasificador y de las políticas de salud (ej: para la 
maternidad un embarazo ectópico y para el pediátrico un recién nacido son, por acuerdo, códigos rojo). Este es un sistema de organización fácil de entender y ha dado buenos resultados en la practica.

Para agilizar el inicio del tratamiento quirúrgico, mientras algunos cirujanos acuden al área de tratamiento de código rojo, otros podrían ir directamente al quirófano y esperar los casos ya definidos vestidos y listos para operar.

Como la mayor demanda quirúrgica recae en cirugía general y ortopedia, el resto de los cirujanos deberá tener conocimientos mínimos de estas especialidades tanto para ayudarlos en caso de gran demanda como para hacer pequeñas intervenciones, proceso llamado "cirujanización del personal".

Las epidemias, deben verse también como un desastre y las instituciones deben trabajar acorde a ello, en tal sentido, no se puede manejar una epidemia con un hospital trabajando normalmente y menos si son pocos en el territorio. Se señala que para ello, la actividad quirúrgica, sistemas de guardia, criterios de ingreso, tareas del personal medico quirúrgico, y otras, se modifican; se suspende la cirugía electiva y la consulta externa y se establece un orden escalonado de ingresos por salas según la epidemia avance. Cada entidad debe tener un protocolo de manejo basado en las mejores evidencias y con el consenso de los grupos de epidemiología.

5. El proceso de evacuación y expansión de los servicios debe ser chequeado periódicamente, en la practica solo hemos tenido necesidad de reagrupar los ingresados para dejar una o más salas para accidentados, pero ante un gran volumen de casos, es necesario optimizar al máximo el espacio y los recursos.

La evacuación de niños pequeños y discapacitados aunque no estén graves, tiene sus particularidades, no se deben enviar a casa sin coordinar con el área de salud para su seguimiento.

Si bien es importante establecer políticas para ganar quirófanos en especial para intervenciones menores en la fase recuperativa, también se deben aprovechar al máximo las capacidades existentes, por ejemplo, los turnos quirúrgicos se alargan cuanto tiempo sea necesario, se crearán por tanto, dos equipos quirúrgicos ante jornadas extenuantes, etc. Para estas situaciones y principalmente la guerra, existen normas tanto en lo referente al personal como los recursos.

6. Los hospitales en situación de una gran demanda de atención a víctimas, no cuentan con algunos recursos como camillas, es necesario buscar alternativas así como variantes para la comunicación. Las personas en su movimiento por la institución como parte de recorridos e inspecciones pueden estar pobremente localizables en un momento en que son necesarias para la toma de decisiones o la ejecución de tareas.

Existen planes de emergencia hospitalaria, muchos de ellos validados por la vida, pero posibles de mejorar; en tal sentido, es acuerdo de esta comisión que el grupo de recomendaciones aquí expresadas, sean enviadas a los decisores e instituciones encargadas de su perfeccionamiento.

La comunidad debe integrarse a los planes de las instituciones cercanas, en especial en zonas rurales.

Destacar que como parte del proceso de compatibilización en el diseño de las instituciones, se debe incluir servicios de urgencia con flujos adecuados de pacientes, que permitan el movimiento de pacientes en camillas. 
Para cualquier situación es necesario trabajar en la reducción de la vulnerabilidad, en especial de los sistemas vitales y la logística de las instituciones, para estar mejor preparados tanto ante fenómenos naturales como accidentes cotidianos.

\section{PRI NCI PALES RECOMENDACI ONES DEL TALLER}

Tras el análisis por cada comisión, se realizó un trabajo de búsqueda de consenso sobre los principales temas y conclusiones tratadas por separado. Ello se presentó nuevamente al panel para su aprobación, y son las principales recomendaciones:

- Es necesario generar encuentros como estos para incrementar el intercambio entre las instituciones, con aquellos que tienen la autoridad para decidir y profesionales encargados del estudio y la preparación del sistema de salud para el enfrentamiento a eventos principalmente de inicio súbitos (de metodología, criterios generales, organización, formas de solucionar problemas, modalidades de trabajo, y otros).

- Necesidad de incrementar tanto la intersectorialidad (relación con otros sectores, principalmente grupos de rescate, Cruz Roja, bomberos, policía, entre otros), como la intrasectorialidad (colaboración entre hospitales, policlínicos y EMM que nos permitan conocer las funciones, necesidades y recursos de cada uno y facilite la actividad como sistema); la integración es un tema medular para que la respuesta sea efectiva y menos costosa.

- Extensión del proceso de capacitación. Aunque se están haciendo cambios curriculares para las carreras de ciencias médicas, es necesario perfeccionarlos, incluirlo en la enseñanza de posgrado y utilizar la misma terminología y metodología para todo el sistema. En tal sentido, se debe mantener la realización de simulacros y simulaciones.

- Necesidad de recursos, muy especialmente medios de comunicación tanto para ambulancias como los sistemas de guardia de las instituciones, ello limita la rapidez y efectividad de la respuesta. Se debe tener sistemas alternativos para la localización del personal.

- Aprobar con las modificaciones propias para cada institución, la implementación del "sistema de tarjetas de funciones" para los servicios de urgencia.

Finalmente se concluye que las experiencias expuestas resultaron sumamente positivas y muy enriquecedoras en todo sentido. Las respuestas de las comisiones y recomendaciones de este taller forman parte del proceso de preparación de los servicios de urgencia y de las instituciones de salud ante las diferentes variantes de situaciones de desastres. No representan una norma a aplicar, pero sí representan una guía que permite la revisión de los planes ante estos eventos, a los que cada unidad le hará las adecuaciones pertinentes y tomará los aspectos que le sean más afines. El sistema por tarjetas de funciones, aprobado por todos, sirve metodológicamente a la respuesta en al menos dos aspectos; el primero en función de la actualización permanente del personal muchas veces cambiante de estas unidades y el segundo, por constituir una versión resumida del plan de estas instituciones. 
Recibido: 21 de noviembre de 2007.

Aprobado: 4 de enero de 2008.

Víctor Rene Navarro Machado. Hospital General Universitario "Dr. Gustavo Aldereguía Lima". Cienfuegos. Avenida 5 de septiembre y calle 31. CP 55100. Cienfuegos, Cuba.

* Relatoría de un taller efectuado el 29 de septiembre de 2007 en Cienfuegos, Cuba. -serif" size="2">Recibido: 21 de noviembre de 2007.

Aprobado: 4 de enero de 2008.

Víctor Rene Navarro Machado. Hospital General Universitario "Dr. Gustavo Aldereguía Lima". Cienfuegos. Avenida 5 de septiembre y calle 31. CP 55100. Cienfuegos, Cuba.

* Relatoría de un taller efectuado el 29 de septiembre de 2007 en Cienfuegos, Cuba.

Anexo

Historia clínica para situaciones de desastres

\begin{tabular}{|c|c|c|c|c|c|c|c|c|c|}
\hline \multicolumn{10}{|c|}{$\begin{array}{c}\text { Sistema Integrado de Urgencias Médicas } \\
\text { Historia clínica para desastres }\end{array}$} \\
\hline \multicolumn{10}{|c|}{ Datos generales } \\
\hline \multicolumn{8}{|l|}{ Nombre: } & $\begin{array}{l}\text { Desconoc } \\
\text { ido }\end{array}$ & \\
\hline Sexo: & Masc & Fem & $\begin{array}{l}\text { Eda } \\
\text { d: }\end{array}$ & Años & $\begin{array}{l}\text { Mes } \\
\text { es }\end{array}$ & \multicolumn{2}{|l|}{ Desconocido } & Fecha: & \\
\hline Incidente & \multicolumn{3}{|c|}{ Municipio } & \multicolumn{4}{|c|}{ Localidad: } & Hora: & \\
\hline \multicolumn{10}{|c|}{ Tipo de Incidente: } \\
\hline \multicolumn{10}{|c|}{ Área de triage } \\
\hline Prioridad: & $\begin{array}{l}\text { Roj } \\
0\end{array}$ & \multicolumn{2}{|c|}{ Amarillo } & \begin{tabular}{l|l} 
Verd \\
e
\end{tabular} & \multicolumn{2}{|c|}{ Negro } & Hora: & \multicolumn{2}{|c|}{ Paciente No: } \\
\hline $\begin{array}{l}\text { Asistencia } \\
\text { previa: }\end{array}$ & & \begin{tabular}{|ll}
$\mathrm{S}$ & No \\
$\mathrm{i}$ &
\end{tabular} & & Probler & mas pr & incipales: & & & \\
\hline
\end{tabular}




\begin{tabular}{|c|c|c|c|c|c|c|c|c|c|c|}
\hline $\begin{array}{l}\text { Respons } \\
\text { able }\end{array}$ & \multicolumn{5}{|c|}{ Nombre: } & \multicolumn{2}{|l|}{ Calificación: } & \multicolumn{3}{|l|}{ Firma: } \\
\hline \multicolumn{11}{|c|}{ Puesto médico de avanzada } \\
\hline \multicolumn{2}{|c|}{ Parámetros } & \begin{tabular}{|l|} 
Hor \\
$a$
\end{tabular} & Hora & Hora & $\begin{array}{l}\text { Hor } \\
\text { a }\end{array}$ & \multicolumn{5}{|c|}{ Procederes generales } \\
\hline \multicolumn{2}{|l|}{ Hora } & & & & & Vendaje & \multicolumn{2}{|c|}{ Oxigeno\% } & \multicolumn{2}{|c|}{ ECG } \\
\hline \multicolumn{2}{|c|}{$\begin{array}{l}\text { Frecuencia } \\
\text { respiratoria }\end{array}$} & & & & & \begin{tabular}{|l|} 
Inmoviliza \\
Ción
\end{tabular} & \multicolumn{2}{|c|}{ Aspiración } & \multicolumn{2}{|c|}{$\begin{array}{l}\text { Reanimaci } \\
\text { ón }\end{array}$} \\
\hline \multicolumn{2}{|c|}{$\begin{array}{l}\text { Insuficiencia } \\
\text { respiratoria }\end{array}$} & & & & & \begin{tabular}{|l|} 
Collarín \\
Cervical
\end{tabular} & \multicolumn{2}{|c|}{ Intubación } & \multicolumn{2}{|c|}{$\begin{array}{l}\text { Cardiover } \\
\text { sion }\end{array}$} \\
\hline \multicolumn{2}{|l|}{$\mathrm{SaO}_{2}$} & & & & & $\begin{array}{l}\text { Tabla } \\
\text { espinal }\end{array}$ & \multicolumn{2}{|c|}{ Ventilación } & \multicolumn{2}{|c|}{$\begin{array}{l}\text { Defibrilaci } \\
\text { on }\end{array}$} \\
\hline \multicolumn{2}{|c|}{\begin{tabular}{|l|}
$\begin{array}{l}\text { Frecuencia } \\
\text { cardiaca }\end{array}$ \\
\end{tabular}} & & & & & $\begin{array}{l}\text { Sonda } \\
\text { uretral }\end{array}$ & \multicolumn{2}{|c|}{ Aerosolterapia } & \multicolumn{2}{|c|}{ Otros } \\
\hline \multicolumn{2}{|c|}{ Presión arterial } & & & & & Sonda & \multicolumn{2}{|c|}{$\begin{array}{l}\text { levine } \\
\text { Monitorización }\end{array}$} & & \\
\hline \multicolumn{2}{|c|}{ Sangramiento } & & & & & \begin{tabular}{|l|} 
Fluidos \\
endovenos \\
os
\end{tabular} & \multicolumn{2}{|c|}{ Volumen: } & \multicolumn{2}{|c|}{ Hora } \\
\hline \multicolumn{11}{|c|}{$\begin{array}{l}\text { Mejor respuesta } \\
\text { ocular }\end{array}$} \\
\hline \multicolumn{11}{|c|}{$\begin{array}{l}\text { Mejor respuesta } \\
\text { verbal }\end{array}$} \\
\hline \multicolumn{11}{|c|}{$\begin{array}{l}\text { Mejor respuesta } \\
\text { motora }\end{array}$} \\
\hline \multicolumn{2}{|c|}{$\begin{array}{l}\text { Escala coma } \\
\text { Glasgow }\end{array}$} & & & & & \begin{tabular}{|l|} 
Medicación \\
administra \\
da
\end{tabular} & \multicolumn{2}{|l|}{ Dosis } & Vía & Hora \\
\hline $\begin{array}{l}\text { Trauma S } \\
\text { revisado }\end{array}$ & core & & & & & & & & & \\
\hline Reacción & pupilar & $\begin{array}{l}00 \\
0\end{array}$ & 000 & 000 & $0 \circ O$ & & & & & \\
\hline ASC quem & nada & & & & & & & & & \\
\hline Código & & & & & & & & & & \\
\hline $\begin{array}{l}\text { Respons } \\
\text { able }\end{array}$ & Nombre & & & & & & $\begin{array}{l}\text { Calificaci } \\
\text { ón: }\end{array}$ & Firma: & & \\
\hline & & & & Are & ea de & evacuación & & & & \\
\hline Paráme & etros & \begin{tabular}{|l|} 
Hor \\
a
\end{tabular} & Hora & Hora & $\begin{array}{l}\text { Hor } \\
\text { a }\end{array}$ & Procederes & generales & & & \\
\hline Hor & & & & & & & & & & \\
\hline $\begin{array}{l}\text { Frecuenci } \\
\text { respirator }\end{array}$ & & & & & & & & & & \\
\hline $\mathrm{SaO} 2$ & & & & & & & & & & \\
\hline $\begin{array}{l}\text { Frecuenci } \\
\text { cardiaca }\end{array}$ & & & & & & & & & & \\
\hline Presión ar & rterial & & & & & \begin{tabular}{|l|} 
Medicación \\
administra
\end{tabular} & Dosis & & Vía & Hora \\
\hline
\end{tabular}




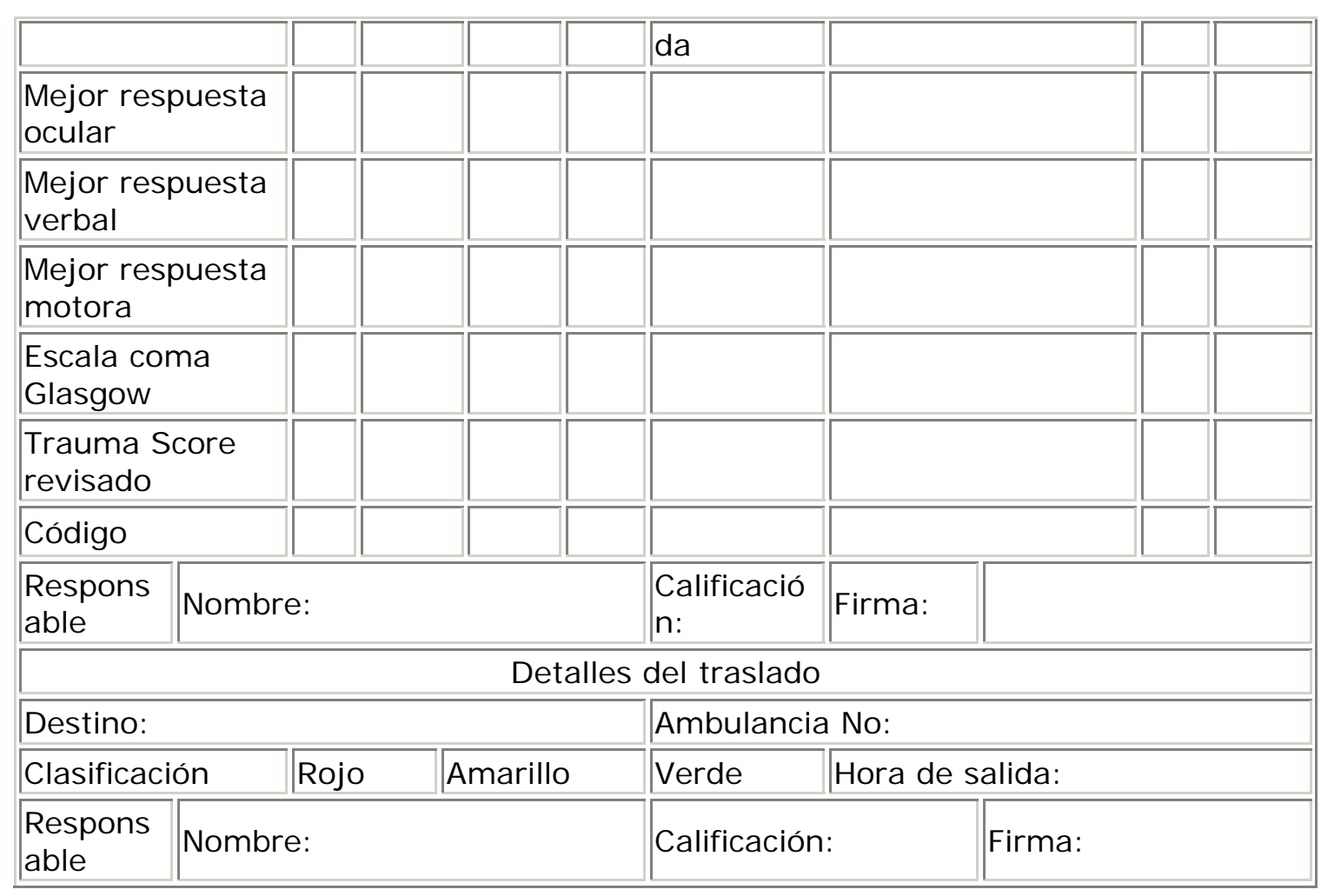

